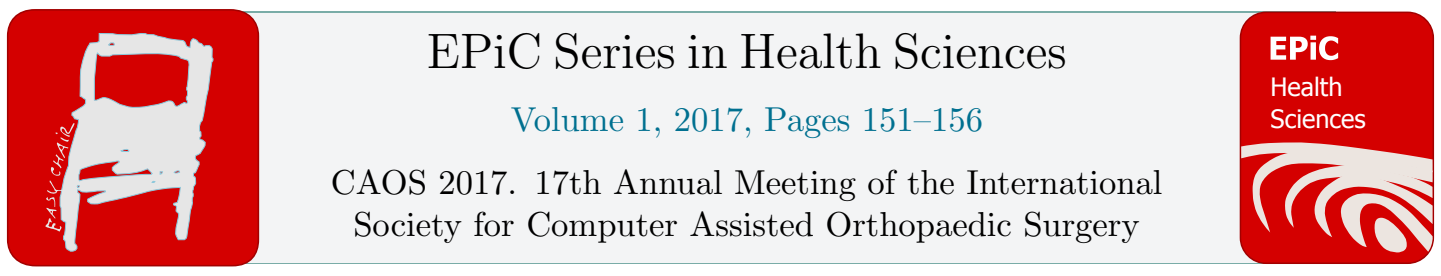

\title{
EVALUATION OF THE USE OF ARTIFICIAL X-RAYS FOR EDUCATIONAL AND INTRAOPERATIVE GUIDANCE DURING C- ARM POSITIONING
}

\author{
Michèle Touchette BASc ${ }^{1}$, Carolyn Anglin, $\mathrm{PhD}^{2}$, Pierre Guy MD MBA ${ }^{3}$, Meena Amlani EdD ${ }^{4}$, \\ Antony Hodgson $\mathrm{PhD}^{5}$ \\ 1,5,* Department of Mechanical Engineering, University of British Columbia, Vancouver, V6T \\ 1Z4, Canada, mtouchette2@gmail.com \\ ${ }^{2}$ Department of Civil Engineering, University of Calgary, Calgary, T2N 1N4, Canada \\ ${ }^{3}$ Department of Orthopaedics, University of British Columbia, Vancouver, V5Z 1M9, Canada \\ ${ }^{4}$ Medical Radiology, British Columbia Institute of Technology, Burnaby, V5G 3H2, Canada
}

\section{INTRODUCTION}

Fluoroscopic C-arms are operated by medical radiography technologists (RTs) in the Canadian operating room (OR). While they do receive formal, accredited training, most of this training is theoretical, rather than hands-on. During their first encounters in the OR, new RTs can experience difficulty achieving the radiographic views required by surgeons, often needing several scout X-rays during C-arm positioning before achieving the correct anatomical view. Furthermore, ambiguous language by surgeons often inadequately conveys their request (Pally 2013). The result is often frustration, unnecessary radiation exposure, and added OR time (Booij 2007).

Several groups have tried to address this problem by overlaying artificial X-ray images on a live video feed (Chen 2013, Reaungamornat 2012, Müller 2011). Others have used artificial X-rays for simulation training (Bott 2008, Gong 2014, Cleary 2004). Though the intent is to improve C-arm positioning accuracy and efficiency during orthopaedic procedures, these systems have primarily been evaluated on 
Evaluation of the Use of Artificial X-Rays for Educational and Intraoperative ... M. Touchette et al.

system accuracy and not on their potential to decrease radiation exposure inside the OR or C-arm positioning time.

The purpose of this study was therefore to evaluate the value of artificial X-rays in enhancing C-arm positioning performance using inexperienced users.

\section{MATERIALS AND METHODS}

We developed an Artificial X-ray Imaging System (AXIS) that generates Digitally Reconstructed Radiographs (DRRs), or artificial X-ray images, based on the relative position of a C-arm fluoroscopy machine and a manikin; the C-arm position was measured using a Northern Digital Inc. Optotrak optical tracking system. Following nominal alignment of the manikin with its CT volume, the DRRs were generated with a Matlab routine (Figure 1) based on Siddon's ray-casting method (Siddon 1985 ) and the runtime was approximately $0.5 \mathrm{~s}$.

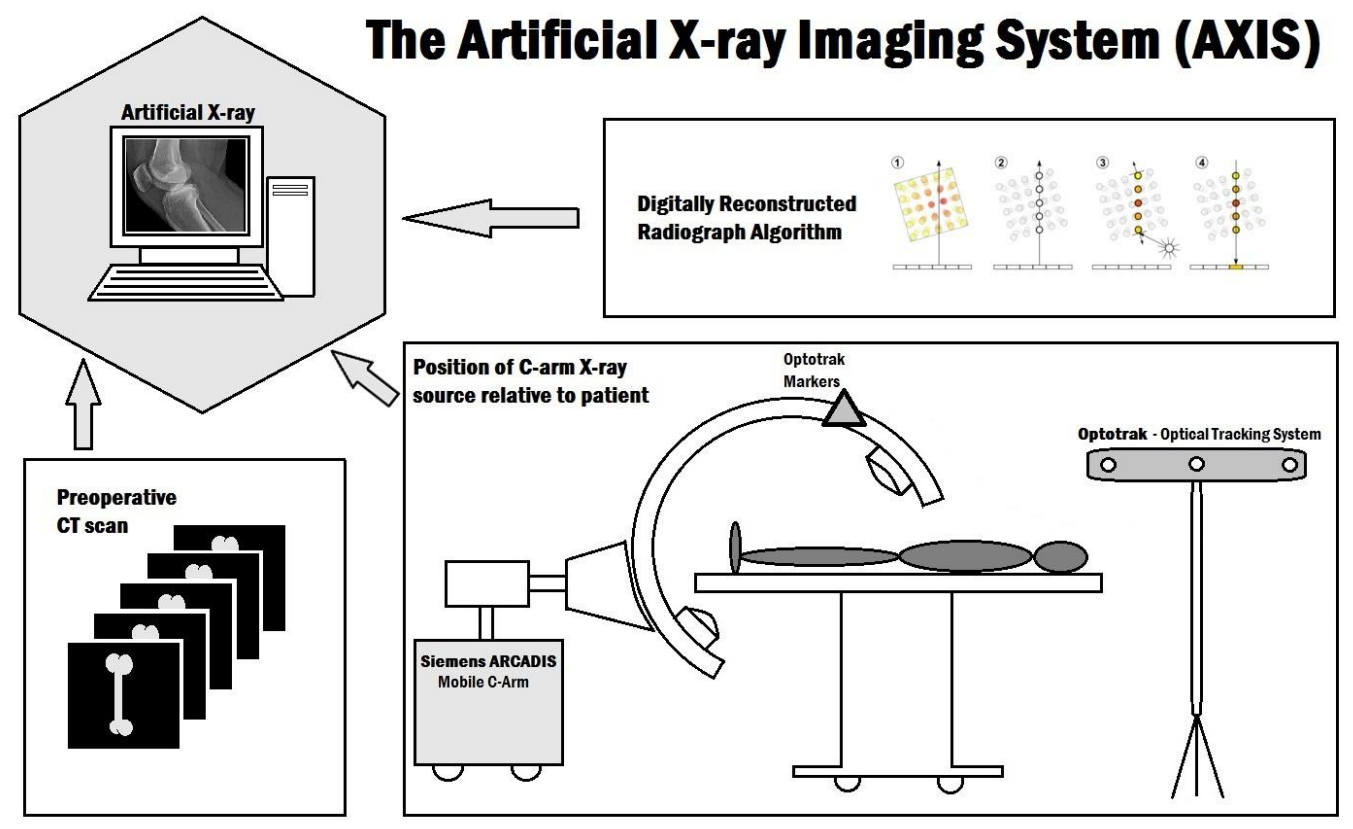

Figure 1: The main elements of the Artificial X-ray Imaging System (AXIS)

For the user study, 30 participants were enrolled and performed four activities: an introduction session, a set of AXIS-guided C-arm positioning tasks, a non-AXISguided task set, and a questionnaire. The main goal of the study was to assess C- 
arm positioning performance with and without AXIS guidance. At the start of each evaluation, the participants were shown a 'casebook' - a set of three target X-ray images that they had to replicate by taking real radiographs of the manikin with the C-arm. Each set of three images was different for the AXIS and non-AXIS tasks, both sets consisting of one easy, one medium, and one difficult radiographic view of the pelvis (as judged by our consulting surgeon). These target images were acquired by an experienced orthopaedic trauma surgeon at Vancouver General Hospital and the corresponding poses measured using the Optotrak system. During the AXISsupported task, artificial X-rays were generated at $2 \mathrm{~Hz}$ and projected on a monitor for guidance, while in the non-AXIS evaluation, the participants had to acquire real scout $\mathrm{X}$-rays to guide them toward the correct view. The task order was randomized. For each imaging task the number of real X-rays and time required per task was recorded, and the C-arm's pose was tracked and compared to the target pose to determine positioning accuracy. In order to provide clinical context to the participants' performance, two orthopaedic surgeons also carried out the non-AXIS evaluation for all six target $\mathrm{X}$-ray images.

Following each session, the number of X-rays, time taken, and accuracies achieved were averaged across the three C-arm imaging tasks for each participant and condition. Hypothesis testing on the means and paired t-tests were carried out using a significance level of $\alpha=0.05$.

\section{RESULTS}

The overall results are summarized in Figure 2. On average, users took significantly fewer real scout X-ray images ( $53 \%$ fewer ( 2.8 vs 6.0 ), $p<0.001$ ) when guided by AXIS. Lateral distance accuracy was improved by $10 \%$ for final $\mathrm{C}$-arm positions and by $26 \%$ for the most accurate intermediate $C$-arm positions when guided by AXIS $(p<0.05)$. There was no significant difference in average task time or angular accuracies between the AXIS and non-AXIS evaluations. Experts were notably faster than the participants in completing the tasks ( 2 min vs $8 \mathrm{~min}$ ), but took more scout images (8 vs 6). Experts tolerated slightly larger lateral differences when reproducing target images (insufficient data to draw statistical conclusions), but seemed to produce somewhat more accurate final images in terms of angular deviations (about $4^{\circ}$ vs about $6.5^{\circ}$ ). 


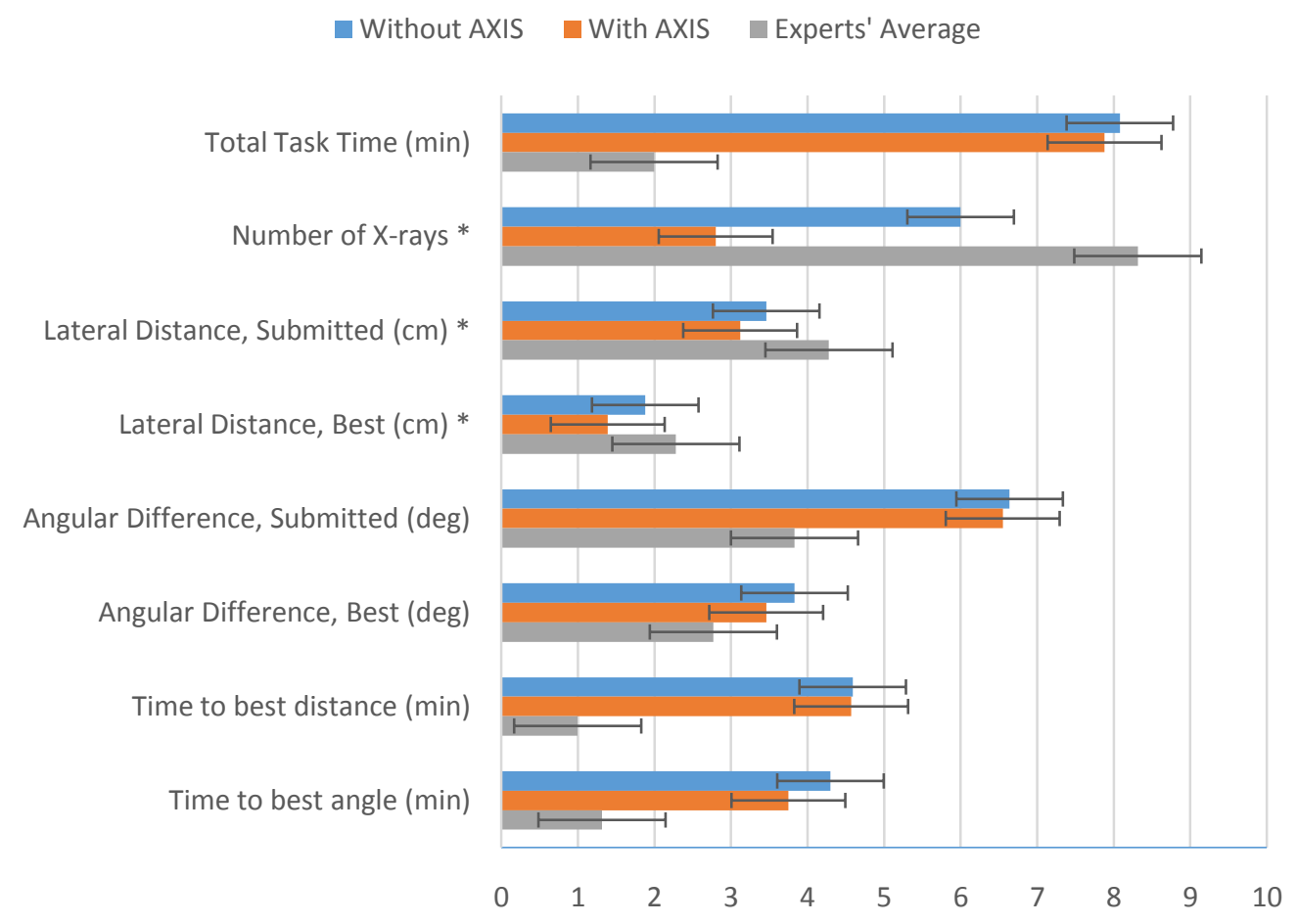

Figure 2: C-arm Imaging Mean Performance Results (* indicates significant differences)

\section{DISCUSSION}

The AXIS system significantly reduced the need for scout X-rays by providing continuously updated visual feedback during C-arm positioning, which could significantly decrease radiation exposure to the patient and OR team. We did not find any significant reduction in task times, but this is perhaps not surprising; when participants were attempting to move the C-arm towards the anatomical goal, they would often experiment with motions of the $\mathrm{C}$-arm to gain immediate feedback on how these affected the image. What time was saved from having to acquire and assess individual scout $\mathrm{X}$-rays seemed instead to have been spent experimenting with AXIS. In practice, surgeons would be able to provide additional feedback and suggestions as to how to adjust the currently-predicted shot in order to optimize the view. Interestingly, participants tended to submit final images that had larger angular deviations than intermediate images they had acquired. Again, in practice the surgeon would likely inform them that the acquired image was adequate for 
use. By not lengthening the positioning task, AXIS is more likely to be accepted for use in the operating room in future.

Our finding of reduced use of scout X-rays when using the AXIS system is compatible with similar findings reported by other groups (e.g., $28 \%$ and $90 \%$ reductions for the CamC and zero-dose navigation systems, respectively - Wang 2010, Müller 2011). Our finding of no significant change in task time with AXIS is somewhat different compared with findings for the virtX system (24\% decrease) and the zero-dose navigation system (68\% increase) (Bott 2008, Müller 2011). Carm positioning accuracy was not reported for these related systems. Overall, we are encouraged by these findings and plan to further develop this system with the goal of deploying it both for training and intraoperative uses.

\section{REFERENCES}

Booij, L, Conflicts in the operating theatre, Current Opinion in Anesthesiology, 20, pp. 152-156, 2006.

Bott O, Teistler M, Duwenkamp C, Wagner M, Marschollek M, Plischke M, Raab B, Stürmer K, Pretschner D, Dresing Klaus, virtX - Evaluation of a computer-based training system for mobile C-arm systems in trauma and orthopaedic surgery, Methods of Information in Medicine, 47(3), pp. 270-278, 2008.

Chen, X, Wang, L, Fallavollita, P, Navab, N, Precise X-ray and video overlay for augmented reality fluoroscopy, International Journal of Computer Assisted Radiology and Surgery, 8, pp. 29-38, 2013.

Cleary, K, Ibanez, L, Ranjan, S, Blake, B, IGSTK: a software toolkit for image-guided surgery applications, International Congress Series, 1268, pp. 473-479, 2004.

Gong, R, Jenkins, B, Sze, R, Yaniv, Z, A Cost Effective and High Fidelity Fluoroscopy Simulator using the Image-Guided Surgery Toolkit (IGSTK), Proceedings of SPIE, 9036, 2014.

Müller, M, Belei, P, de la Fuente, M, Strake, M, Weber, O, Burger, C, Radermacher, $K$, Wirtz, D, Evaluation of a fluoroscopy-based navigation system enabling a virtual radiation-free preview of X-ray images for placement of cannulated hip screws, $A$ cadaver study, Computer Aided Surgery, 16(1), pp. 22-13, 2011. 
Pally E, Kreder HJ, Survey of terminology used for the intraoperative direction of Carm fluoroscopy, Canadian Journal of Surgery, 56(2), pp. 109-112, 2013.

Reaungamornrat, S, Otake, Y, Uneri, A, Schafer, S, Mirota, D, Nithiananthan, S, Stayman, J, Kleinszig, G, Khanna, A, Taylor, R, Siewerdsen, J, An on-board surgical tracking and video augmentation system for $\mathrm{C}$-arm image guidance, International Journal of Computer Assisted Radiology and Surgery, 7, pp. 647-665, 2012.

Siddon R, Fast calculation of the exact radiological path for a three-dimensional CT array, Medical Physics, 12(2), pp. 252-255, 1985.

Wang L, Weidert S, First animal cadaver study for interlocking of intramedullary nails under camera augmented mobile C-arm, A surgical workflow based preclinical evaluation, Proceedings of IPCAI, Lecture Notes in Computer Science, 6135, pp. 5666, 2010. 